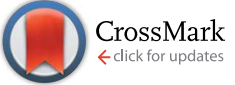

Cite this: RSC Adv., 2017, 7, 7203
Received 12th November 2016 Accepted 11th January 2017

DOI: 10.1039/c6ra26693b

www.rsc.org/advances

\section{Benefits of tubular morphologies on electron transfer properties in CNT/TiNT nanohybrid photocatalyst for enhanced $\mathrm{H}_{2}$ production}

\author{
M. Mamatha Kumari, ${ }^{\star a b}$ A. Priyanka, ${ }^{a}$ B. Marenna, ${ }^{a}$ Prathap Haridoss, ${ }^{\text {*b }}$ \\ D. Praveen Kumar and M. V. Shankar ${ }^{a}$
}

In order to study the influence of one dimensional tubular structures for effective electron and hole transportation onto the surface of a photocatalyst leading to efficient solar photocatalytic hydrogen production, functionalized carbon nanotube $(\mathrm{FCNT}) / \mathrm{TiO}_{2}$ nanotube nanohybrids were prepared. $\mathrm{TiO}_{2}$ nanotubes (TiNTs) were prepared by hydrothermal method. A series of novel functionalized carbon nanotube/ $\mathrm{TiO}_{2}$ nanotube nanohybrids (CTT) were prepared for different wt\% (1-20) of FCNTs by wet impregnation method to extend absorption in the visible region and also to retard the electron-hole pair recombination and thereby to enhance the $\mathrm{H}_{2}$ production capability under solar light irradiation. The functionalized carbon nanotube/ $\mathrm{TiO}_{2}$ nanotube nanohybrids (CTT) were characterized with XRD, TEM, DRS-UV-Vis, Raman spectroscopy and XPS for crystal structure, morphology, optical properties and chemical composition. Addition of FCNTs to the TiNTs in CTT nanohybrids extended the absorption to the visible region. Relative electron-hole recombination times were measured with photoluminescence spectra. The highest $\mathrm{H}_{2}$ generation of $29904 \mu \mathrm{mol} \mathrm{g}{ }^{-1}$ was observed after $4 \mathrm{~h}$ under optimal conditions due to better separation of electron-hole pairs and electron conducting properties.

\section{Introduction}

Since the discovery of carbon nanotubes by Ijima, ${ }^{1 a}$ they have been of great interest to researchers for their 1D tubular structure, good conductivity, high surface area and unique optical properties $^{1 b, c}$. Due to these interesting properties, they have been used as co-catalyst and also as sensitizer in photocatalytic applications such as photodegradation of organic dyes, removing pollutants from air and water, and hydrogen generation from water splitting etc. ${ }^{2}$ Photocatalysts that generate hydrogen through water splitting by utilizing solar energy, contribute to environmentally friendly programs due to their properties such as zero emissions. Among the photocatalysts, $\mathrm{TiO}_{2}$ is a promising candidate because of its relatively low cost, non-toxicity, stability and suitable band edge positions for redox reactions.

Among several nanocrystalline structures of $\mathrm{TiO}_{2}$ photocatalysts, $\mathrm{TiO}_{2}$ nanotubes (TiNT) have more optimal properties for photocatalytic $\mathrm{H}_{2}$ production due to their one dimensional nanostructure. Previous studies have shown that combination

\footnotetext{
${ }^{a}$ Nanocatalysis and Solar Fuels Research Laboratory, Department of Materials Science \& Nanotechnology, Yogi Vemana University, Kadapa-516 003, Andhra Pradesh, India. E-mail: mamatha@yogivemanauniversity.ac.in; Tel: +91-8886577869

${ }^{b}$ Department of Metallurgical and Materials Engineering, Indian Institute of Technology Madras, Chennai 600 036, Tamil Nadu, India. E-mail: prathap@iitm. ac.in; Tel: $+91-9444468235$
}

of TiNT with other nanomaterials have increased photocatalytic activity. $^{3}$

Several studies ${ }^{4}$ have been carried out to utilize CNTs in improving the photocatalytic $\mathrm{H}_{2}$ production efficiency of $\mathrm{TiO}_{2}$ nanoparticles in view of their unique optical and electron transport properties. Silva et al. ${ }^{5 a}$ studied the photocatalytic $\mathrm{H}_{2}$ production of $\mathrm{MWCNT} / \mathrm{TiO}_{2}$ photocatalyst from aqueous methanol and saccharide solutions. Recently Tarigh et al. ${ }^{5 b}$ reported a simple, effective and recoverable photocatalyst $\mathrm{MWCNT} / \mathrm{TiO}_{2}$ for enhanced photocatalytic activity. There is still a significant potential for improvement in the photocatalytic activity of all the above photocatalysts by reducing the electronhole recombination through one dimensional nanostructure. ${ }^{7}$ This motivated us to study effect of one dimensional nanostructure of both CNTs and TiNTs on the photocatalytic $\mathrm{H}_{2}$ production under solar irradiation.

Also, less attention has been paid to synthesis of CNT/TiNT nanohybrid and its photocatalytic activity. Doong et al. ${ }^{6}$ studied degradation efficiency of CNT/TiNT composite and found that it is a promising nano-adsorbent for coupled removal of organic as well as heavy metal ions in solution. However, to the best of our knowledge research work on CNT/ TiNT ( $\mathrm{TiO}_{2}$ nanotubes) nanohybrid for hydrogen generation under natural solar light by water splitting, is scarce.

In the present work we study the preparation method for CNT/TiNT nanohybrid and its hydrogen generation efficiency under natural solar light irradiation by water splitting. 


\section{Experimental methods}

Materials

All the chemicals used for the present work are of analytical grade and the details of the chemicals and catalysts used are given below. Commercial titanium dioxide $\mathrm{TiO}_{2} \mathrm{P}-25$ catalyst (anatase $80 \%$, rutile $20 \%$, surface area $=50 \mathrm{~m}^{2} \mathrm{~g}^{-1}$ and particle size $27 \mathrm{~nm}$ ) was received from Degussa Corporation, Germany. Commercial $\mathrm{TiO}_{2}$ (particle size $\approx 150 \mathrm{~nm}$, surface area $=5.1 \mathrm{~m}^{2}$ $\left.\mathrm{g}^{-1}, \mathrm{LAB}\right)$ used for nanotube synthesis was purchased from Merck, India. Sodium hydroxide pellets (99\%), hydrochloric acid (35\%), $\mathrm{HNO}_{3}(33 \%), \mathrm{H}_{2} \mathrm{SO}_{4}(38 \%)$ and glycerol (98\%) were received from Merck, India. De-ionized water (100\%, battery grade) used for synthesis, impregnation process and photocatalytic experiments was procured from LIMRA, Kadapa, India.

\section{Synthesis of FCNTs}

Carbon nanotubes were synthesized by arc discharge method in open air. ${ }^{4 e}$ Functionalization of CNTs has been described in detail in an earlier report. ${ }^{4 e}$ Briefly functionalization of CNTs was carried out to remove amorphous carbon, metal catalyst and also to introduce acid functional groups to the carbon nanostructures, by ultrasonicating them in $3: 1$ volume ratio mixture of $\mathrm{H}_{2} \mathrm{SO}_{4} / \mathrm{HNO}_{3}$ at $40{ }^{\circ} \mathrm{C}$ for $2 \mathrm{~h}$, followed by washing with plenty of water till reaches $\mathrm{pH} \approx 7$ and drying at $80{ }^{\circ} \mathrm{C}$ overnight.

\section{Synthesis of TiNTs}

$\mathrm{TiO}_{2}$ nanotubes (TiNTs) were prepared by alkaline hydrothermal method. ${ }^{4 f}$ Briefly, the synthesis process involves dispersion of $2.5 \mathrm{~g}$ of $\mathrm{TiO}_{2}$ (Merck) in $10 \mathrm{M} \mathrm{NaOH}$ solution and cooking in $250 \mathrm{~mL}$ Teflon lined stainless steel autoclave at 100$130{ }^{\circ} \mathrm{C}$ for $20-24 \mathrm{~h}$. The white slurry obtained was then washed with distilled water, later by dilute $\mathrm{HCl}$, finally washed with ethanol and dried at $80{ }^{\circ} \mathrm{C}$. Dried powder was subjected to calcination at $350{ }^{\circ} \mathrm{C}$ for $3 \mathrm{~h}$ to get TiNT.

\section{Synthesis of FCNTs/TiNTs nanohybrid}

$\mathrm{TiO}_{2}$ nanotubes/FCNT nanohybrid photocatalysts were prepared through wet impregnation method. Functionalized CNTs (FCNTs) and hydrothermally synthesized TiNTs were taken as starting materials. In a typical impregnation procedure, required amount (1-20 wt\%) of FCNTs (Table 1) was dispersed in ethanol aqueous solution in beaker-1,

Table 1 Photocatalysts used

\begin{tabular}{ll}
\hline Photocatalyst & Nomenclature \\
\hline $\begin{array}{l}\text { Functionalized carbon } \\
\text { nanotubes }\end{array}$ & FCNT \\
$\mathrm{TiO}_{2}$ nanotube & TiNT \\
$1 \mathrm{wt} \%$ FCNT loaded TiNT & CTT-1 \\
$2 \mathrm{wt} \%$ FCNT loaded TiNT & CTT-2 \\
$10 \mathrm{wt} \%$ FCNT loaded TiNT & CTT-10 \\
$20 \mathrm{wt} \%$ FCNT loaded TiNT & CTT-20
\end{tabular}

ultrasonicated and magnetically stirred for $1 \mathrm{~h}$. Simultaneously, in beaker-2, TiNT was dispersed in ethanolic aqueous solution. Then contents in beaker-1 was transferred into beaker- 2 and resulting mixture was ultrasonicated for $1 \mathrm{~h}$. The TiNT/FCNT mixture containing ethanolic aqueous solvent, was subjected to heating and magnetic stirring till the solution is completely evaporated. Nanohybrid mixture was kept for drying in oven at $80{ }^{\circ} \mathrm{C}$ overnight and calcination in tubular furnace in air at $450{ }^{\circ} \mathrm{C}$ for $2 \mathrm{~h}$, with a heating rate of $5{ }^{\circ} \mathrm{C} \mathrm{min}^{-1}$.

\section{Characterization}

Transmission Electron Microscopy (TEM) images were recorded on a PHILIPS instrument $(120 \mathrm{kV})$. The powder catalyst samples for TEM observations were directly deposited onto carbon coated Cu-grids. The XRD patterns were recorded on Siemens D$5000 \mathrm{X}$-ray diffractometer $\left(2 \theta=20-90^{\circ}\right)$. Thermo gravimetric analysis (TGA) was performed on a universal instrument using TGA Q 500 analyzer with a heating rate of $10{ }^{\circ} \mathrm{C} \mathrm{min}^{-1}$ under nitrogen flow. Diffuse Reflectance UV-Visible spectra were recorded on a GBC UV-Visible Cintra 10e spectrometer, in the wavelength range 200-500 $\mathrm{nm}$. X-ray photoelectron spectra (XPS) were recorded on a KRATOS AXIC 165 equipped with Mg $\mathrm{K}_{\alpha}$ radiation. Confocal Raman spectra of all the samples were recorded using Raman spectrometer (RENISHAW) operating with an excitation wavelength of $532 \mathrm{~nm}$ and a power $1 \mathrm{~mW}$. Photoluminescence (PL) spectra were recorded using Spectrofluorometer, (FLUORA MAX 4P) excitation wavelength at $320 \mathrm{~nm}$.

\section{Photocatalytic $\mathrm{H}_{2}$ production activity}

Photocatalytic hydrogen evolution reactions were carried out in a $150 \mathrm{~mL}$ quartz reactor sealed using an air-tight rubber septum to keep the experimental set up leak-proof and enable gas sampling. Typically, $5 \mathrm{mg}$ of catalyst was suspended in $50 \mathrm{~mL}$ of a 5 vol\% glycerol aqueous solution. Prior to solar experiments, the catalyst suspension was stirred in dark for 30 min followed by degassing and purging with nitrogen (high pure) gas to eliminate oxygen from the reaction system. Catalytic activities were performed under continuous magnetic stirring and solar light was used to excite the photocatalyst for red-ox reaction on the catalyst surface. The amount of $\mathrm{H}_{2}$ and $\mathrm{O}_{2}$ produced was measured by offline gas chromatography. Control experiments were carried out under similar experimental conditions in the absence of photocatalyst or sun light, neither $\mathrm{H}_{2}$ nor $\mathrm{O}_{2}$ gas was detected.

\section{Results and discussion}

\section{$\mathrm{X}$-ray diffraction}

Fig. 1 presents the XRD patterns of TiNT, CTT-1, CTT-2, CTT-10, CTT-20 and FCNT photocatalysts. FCNT showed a broad peak located between $20^{\circ}$ and $30^{\circ}$ which is assigned to the (002) plane of the carbon structure. The diffraction peaks of $\mathrm{TiO}_{2}$ were identified with reference to JCPDS database. The photocatalysts TiNT, CTT-1 and CTT-2 displayed diffraction peaks at $2 \theta=$ $25.3^{\circ}, 37.8^{\circ}, 48.0^{\circ}, 53.8^{\circ}, 54.9^{\circ}$ and $62.5^{\circ}$ which are the peaks of 


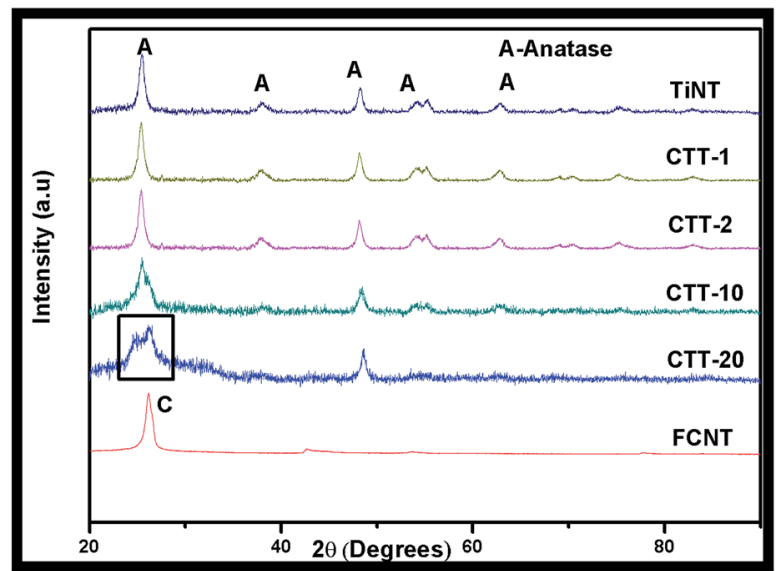

Fig. 1 XRD patterns of TiNT, CTT-1, CTT-2, CTT-10, CTT-20 and FCNTs.

(101), (004), (200), (105), (211) and (204) respectively for anatase phase (JCPDS 21-1272). Also, with increase in FCNT content, a new peak at $26.2^{\circ}$ is observed clearly for CTT-20 corresponding to the carbon (100) in FCNT. Carbon (100) peak is not observed in CTT-2 and slightly in CTT-10, due to the presence of lower amount of FCNT. Kim et al. ${ }^{8 a}$ studied $\mathrm{TiO}_{2}$ nanoparticle loaded on graphene/carbon nanocomposites photocatalysts and XRD pattern displayed characteristic peaks of anatase-rutile phases in addition to carbon nanostructures. We have also observed characteristic XRD peaks of both $\mathrm{TiO}_{2}$ anatase-rutile and carbon in FCNTs $/ \mathrm{TiO}_{2}$ nanoparticles nanohybrid material. ${ }^{4 e}$ The results reveal that addition of FCNTs to $\mathrm{TiO}_{2}$ nanotubes suppressed both grain growth and phase transformation. This is mainly attributed to fine dispersion of $\mathrm{TiO}_{2}$ nanotubes on FCNTs surface.

\section{TEM}

TEM image Fig. 2 shows the as-prepared CTT-2 nanohybrid. Fig. 2(a) shows that bundles of $\mathrm{TiO}_{2}$ nanotubes of lengths 100-
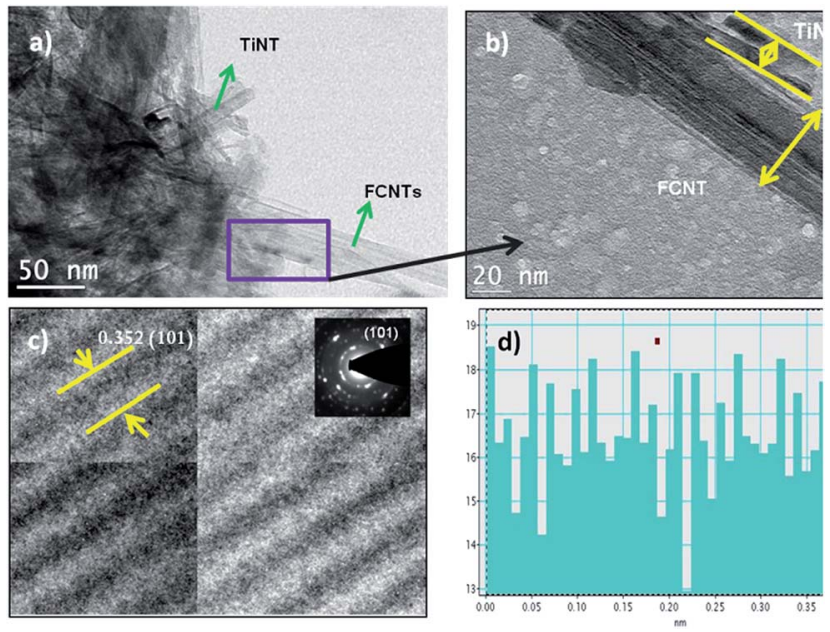

Fig. 2 TEM and HRTEM images of $\mathrm{TiO}_{2}$ nanotube/FCNT nanohybrid (a), (b) showing the morphological structure (c), (d) showing the interplanar spacing and live FFT image respectively.
$500 \mathrm{~nm}$ are dispersed on the FCNTs whose diameters are in the range of $30-50 \mathrm{~nm}$. As both FCNTs and TiNTs are tubular structure, in order distinguish one from the other, rectangle marked region is magnified as shown in Fig. 2(b). Further, HRTEM image Fig. 2(c) showing the lattice spacing of TiNT is subjected to profile of live FFT image Fig. 2(d) to measure the observed interplanar spacing. Measured lattice spacing from live FFT is $0.352 \mathrm{~nm}$ which is approximate to that of (101) anatase $\mathrm{TiO}_{2}$. This is further confirmed by the SAED pattern shown in the inset of Fig. 2(c).

\section{UV-Visible DRS}

Optical properties of the prepared nanohybrids CTT-1, 2,10, 20 \& TiNT were studied by analyzing UV-Visible Diffuse Reflectance spectrum (Fig. 3(a)). It is seen that CTT-1, 2,10 \& 20 showed enhanced absorption towards visible region than TiNT. Approximate absorption edges of CTT-1, 2,10 \& 20 are in the order $395,424,413$ and $449 \mathrm{~nm}$ while that of TiNT is $389 \mathrm{~nm}$. A clear red shift of the absorption lengths can be attributed to presence of FCNTs in the nanohybrid. It is observed that CTT-2 showed higher absorption and absorption edge than CTT-10 and CTT-20. This increased absorption in visible region could also be due to $\pi \rightarrow \pi^{*}$ of CNT and $\mathrm{n} \rightarrow \pi^{*}$ between n-orbit of oxygen species of TiNT. ${ }^{8 b-d}$

Kubelka-Munk plot (Fig. 3(b)) shows that the band gap values of CTT nanohybrids are reduced to $2.98 \mathrm{eV}$ from a value

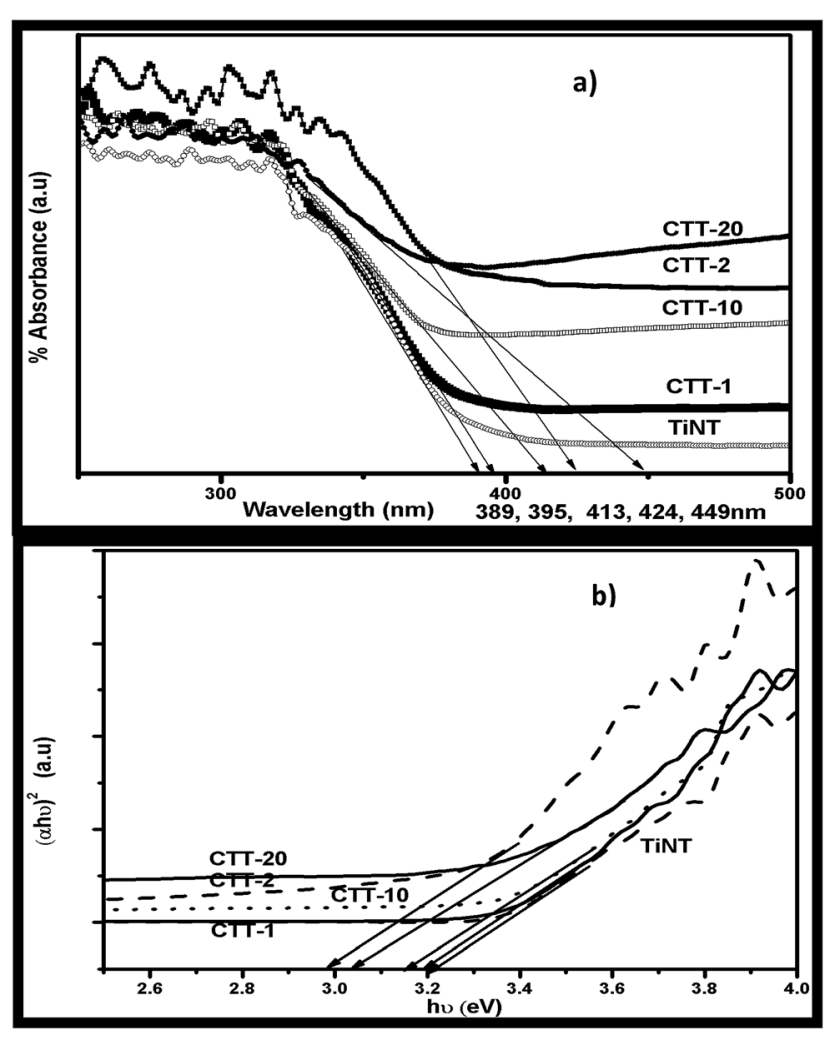

Fig. 3 (a) UV-Vis DRS and (b) transformed Kubelka-Munk functions $(\alpha h \nu)^{2}$ vs. photon energy $(h \nu, \mathrm{eV})$ of $\mathrm{CTT}-1,2,10,20 \mathrm{~g}$ TiNT photocatalysts. 
$3.2 \mathrm{eV}$ of TiNT. This decrease in band gap energies is due to synergetic effect of presence of FCNTs attached to the surface of TiNT rather than occupying lattice sites in the nanohybrid. ${ }^{8 e}$

\section{XPS analysis}

XPS analysis was performed on prepared CTT-2 and the wide scan spectrum (Fig. 4(a)) contains Ti 2p, O 1s and C 1s of $\mathrm{TiO}_{2}$. High resolution XPS of Ti 2p shows (Fig. 4(b)) intense peaks at $459.70 \mathrm{eV}$ and $460.2 \mathrm{eV}$ corresponding to $2 \mathrm{p}_{3 / 2}$ and second highest peak at $465.9 \mathrm{eV}$ corresponding to $2 \mathrm{p}_{1 / 2}$, indicating the presence of $\mathrm{Ti}^{4+}$ in the nanohybrid. Also, these binding energies are slightly shifted from the corresponding original binding energies at $458.7 \mathrm{eV}$ and $464.5 \mathrm{eV}$ in $\mathrm{TiO}_{2}$. Such results were reported earlier indicating the strong interaction between $\mathrm{TiO}_{2}$ nanoparticles and CNTs. ${ }^{\boldsymbol{a}, \boldsymbol{b}} \mathrm{O}$ 1s region is composed of two peaks (Fig. 4(c)). Peaks at BE $530.5 \mathrm{eV}$ and $532.7 \mathrm{eV}$ correspond to bulk oxygen in $\mathrm{Ti}-\mathrm{O}-\mathrm{Ti}$ and surface oxygen from the hydroxyl group $\mathrm{Ti}-\mathrm{OH}$.

XPS of CTT-2 curve has peaks at $285.4 \mathrm{eV}, 286.2 \mathrm{eV}$ and $288.5 \mathrm{eV}$ corresponding to the $\mathrm{C}-\mathrm{O}, \mathrm{C}=\mathrm{O}$ and $-\mathrm{COO}$ (Fig. 4(d)). Binding energy peaks around $286 \mathrm{eV}$ and $288 \mathrm{eV}$ were attributed to $\mathrm{C}-\mathrm{O}$ and $\mathrm{C}=\mathrm{O}$ bonds. This indicates the formation of carbonated structures and probable Ti-O-C bonding. ${ }^{9 c}$ The interaction of TiNT with FCNTs was further studied using Raman analysis.

\section{Raman analysis}

Raman spectrum of CTT-2 is shown in the Fig. 5(a). Characteristic bands at $1326 \mathrm{~cm}^{-1}$ and $1579 \mathrm{~cm}^{-1}$ in CTT-2 corresponds to $\mathrm{D}$ and $\mathrm{G}$ bands of CNTs respectively. The relative intensity ratio of $\mathrm{D}$ band to $\mathrm{G}$ band $\left(I_{\mathrm{D}} / I_{\mathrm{G}}\right)$, known as index of graphitization, is found to be 1.1 for CTT-2 nanohybrid while that of CNTs is 0.7.

This indicates that functionalization in acid mixture and calcination temperature during nanohybrid formation did not significantly alter the CNT structure in the nanohybrid. Dai et al. also reported an $I_{\mathrm{D}} / I_{\mathrm{G}}$ ratio of 0.78 for $\mathrm{MWNT}^{-\mathrm{TiO}_{2}}$, comparable to that of the pure MWNTs (0.70), indicating that the graphitic structure of MWNTs in the nanocomposites did not change much after functionalization in nitric acid or during the hydrothermal treatment. ${ }^{{ }^{d} \boldsymbol{d}}$ The new peak observed at 1616 $\mathrm{cm}^{-1}$ (Fig. 5(b)) is due to the interaction between FCNTs and TiNTs. This is in agreement with reported results. ${ }^{9 e}$

\section{BET surface area}

The surface area of the TP, TiNT and CTT nanohybrid photocatalysts are as shown in the Table 2 . An increase in the surface area of the catalyst with increase in ratio of FCNTs to TiNT, is observed. Generally addition of FCNTs reduces the agglomeration of $\mathrm{TiO}_{2}{ }^{10 a}$ This property of FCNTs ensures the higher surface area of the nanohybrid with increase in FCNTs addition. The decrease in photocatalytic activity of the CTT-10, CTT-20 is due to high surface area where CNTs become recombination centers for the generated electron-hole pairs and is confirmed by the PL spectra.

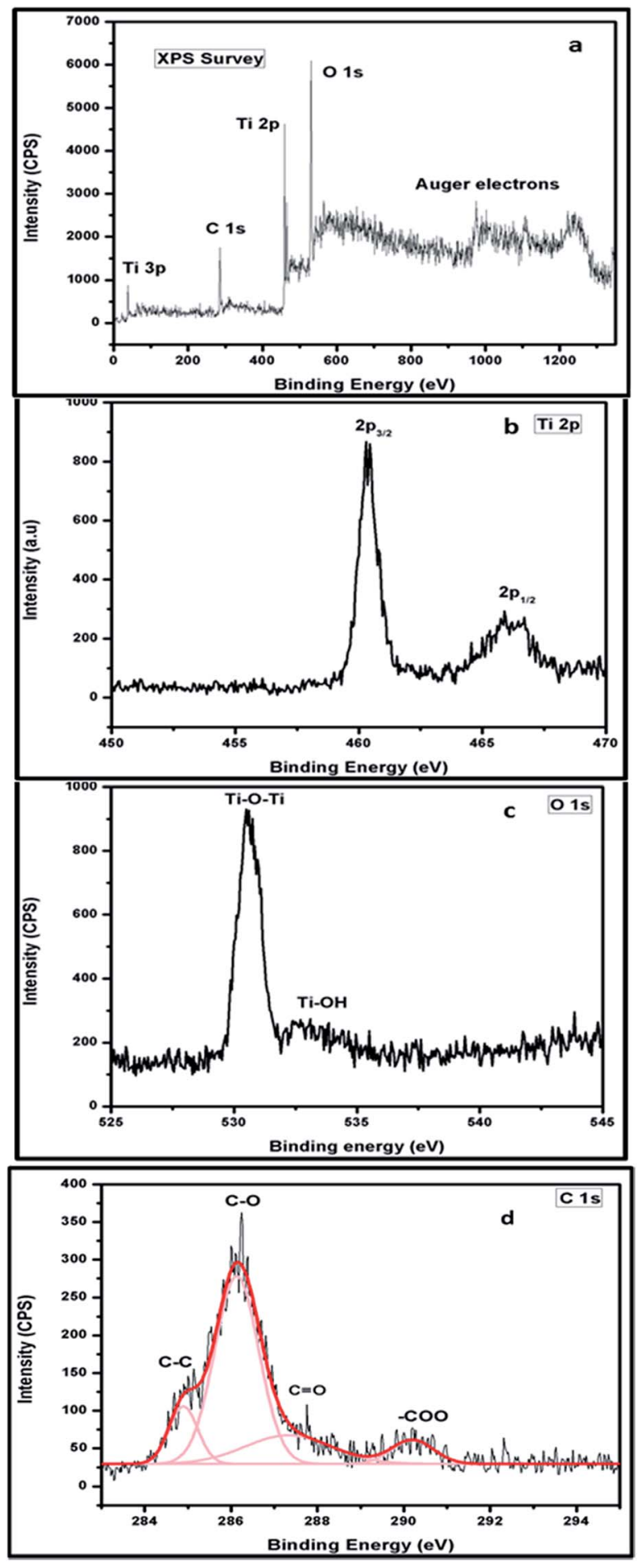

Fig. 4 (a) XPS wide CPS scan (b) Ti 2p (c) O 1s (d) C 1s of CTT-2.

\section{Photoluminescence spectra}

Fig. 6 displays the PL spectra of TiNT, CTT-1, $-2,-10 \& 20$ in the wavelength range of $350-550 \mathrm{~nm}$. TiNT showed broad spectrum centred around $375 \mathrm{~nm}$, whereas the nanohybrid catalysts showed decrease in peak intensity in the following order CTT-1 $<$ CTT-2 < CTT-10 < CTT-20. The CTT-20 catalyst displayed blue shift in PL intensity which is attributed to the decrease in $\mathrm{TiO}_{2}$ 


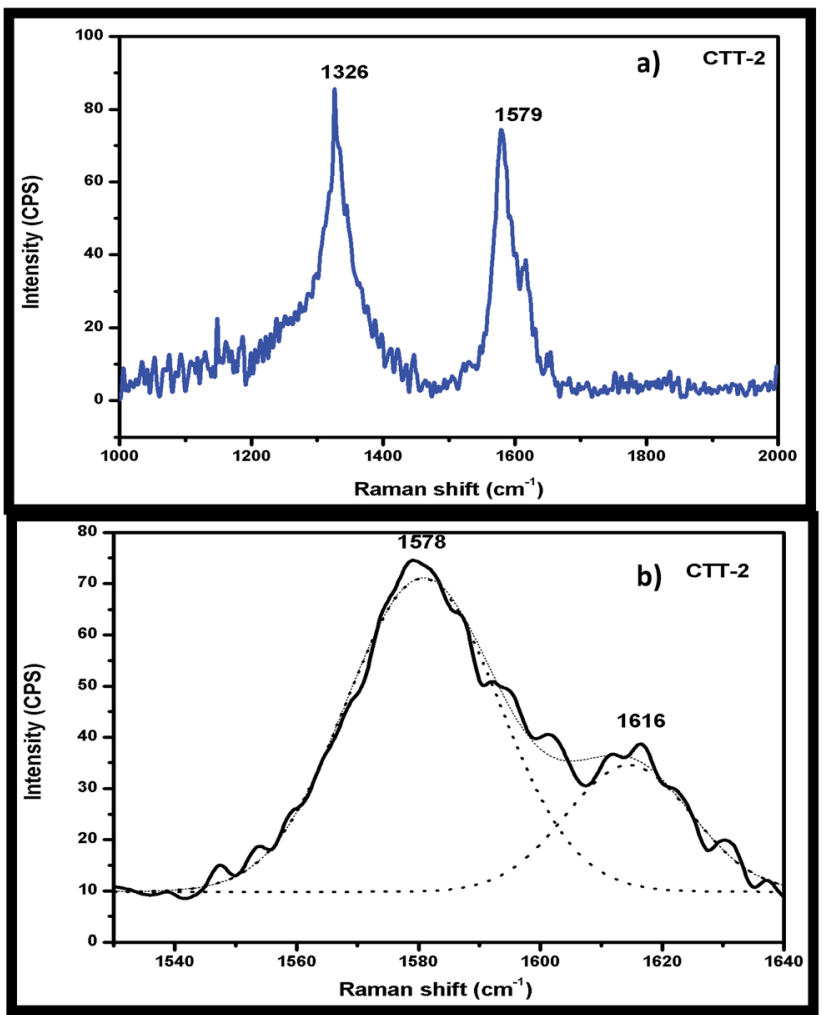

Fig. 5 (a) Raman spectra of CTT-2 catalyst and (b) its expanded view from 1500 to $1640 \mathrm{~cm}^{-1}$.

component in nanohybrid catalyst. These results suggest that drastic reduction of charge carrier recombination with associated reduction of PL-intensity. Both CTT- 1 and CTT-2 have lower peak intensity and hence showed higher rate of $\mathrm{H}_{2}$ production (vide supra). Kim et al. ${ }^{8 a}$ compared the PL intensity of $\mathrm{TiO}_{2}$ /graphene carbon nanocomposites with $\mathrm{TiO}_{2}$ itself, and they explained that strong interaction between excited electrons on $\mathrm{TiO}_{2}$ particles and graphene diminished the peak intensity of nanocomposites, this phenomenon was attributed to decrease in charge carrier recombination.

Praveen Kumar et al., observed decrease in PL peak intensity with $\mathrm{Cu}_{2} \mathrm{O}$ sensitized $\mathrm{TiO}_{2}$ nanorods with nanocavities. They concluded that decrease in peak intensity is due to lower recombination rate of charge carriers localized in nanocavities of $\mathrm{TiO}_{2}$ nanorods. ${ }^{4 f}$ These results are in agreement with earlier reports. ${ }^{10 a, b}$

\section{Photocatalytic $\mathrm{H}_{2}$ production activity}

The photocatalytic performance of the $\mathrm{FCNT} / \mathrm{TiO}_{2}$ nanotube was evaluated by $\mathrm{H}_{2}$ evolution in the presence of $5 \mathrm{vol} \%$ glycerol aqueous solution, which is the sacrificial reagent, under natural solar irradiation. Fig. 7 shows the $\mathrm{H}_{2}$ production activity of TiNT, CTT-1, 2, 10 \& 20 photocatalysts with increase in solar irradiation time. Linear increase in the $\mathrm{H}_{2}$ production activity of all the photocatalysts is observed with increase in time.

$\mathrm{H}_{2}$ production activity is enhanced with the addition of FCNT to TiNT. Also, $\mathrm{H}_{2}$ production activity of CTT- 1 and CTT-2
Table 2 BET surface area of TiNT and CTT photocatalysts

\begin{tabular}{lll}
\hline S. No. & Nanohybrid & SBET $\left(\mathrm{m}^{2} \mathrm{~g}^{-1}\right)$ \\
\hline 1 & CTT-1 & 121.8 \\
2 & CTT-2 & 120.5 \\
3 & CTT-10 & 169.5 \\
4 & CTT-20 & 193.6 \\
5 & TiNT & 70.2 \\
6 & TP & 50
\end{tabular}

showed enhanced and almost similar activity. This enhancement may be attributed to higher surface area of FCNT and better bonding of FCNT and TiNT. $\mathrm{H}_{2}$ production activity of CTT-10 showed lower solar photocatalytic $\mathrm{H}_{2}$ production than unmodified TiNT due to losses in light absorption and electronhole recombination. Among CTT nanohybrids CTT-2 showed highest $\mathrm{H}_{2}$ production of $7476 \mu \mathrm{mol} \mathrm{h} \mathrm{h}^{-1} \mathrm{~g}^{-1}$.

CTT nanohybrid also shows increase in $\mathrm{H}_{2}$ production activity due to FCNT acting as both co-catalyst and sensitizer. However $\left(\mathrm{TiO}_{2}\right.$ nanotubes/FCNTs) CTT samples showed higher activity compared to other $\mathrm{TiO}_{2} / \mathrm{MWCNTs}$ photocatalyst ${ }^{4 a, b, e}$ reported in literature mainly due to the following reasons. Formation of Ti-O-C at the interface of $\mathrm{TiO}_{2}$ and FCNTs favours the effective charge transfer between them. Also, the 1D tubular structures of $\mathrm{TiO}_{2}$ nanotubes, in addition to the FCNTs, which allowed uni-directional flow of electrons, hence reducing electron-hole recombination.

\section{Photocatalytic stability}

Fig. 8 depicts amount of the $\mathrm{H}_{2}$ production tested for $4 \mathrm{~h}$ in 4 cycles of study. These results reveal that almost identical amount of the $\mathrm{H}_{2}$ generation was recorded in the all the four experiments. The minor decrease in the total $\mathrm{H}_{2}$ generation was observed in $3^{\text {rd }}$ and $4^{\text {th }}$ cycles which can be attributed to the poor surface interaction of reaction intermediates formed during oxidation of glycerol in solution. These intermediates could have been adsorbed onto the surface of the photocatalyst which influenced the light penetration to the surface and resulted decrease in the rate of the $\mathrm{H}_{2}$ production. The stability of TiNTs under recycling conditions was reported elsewhere for

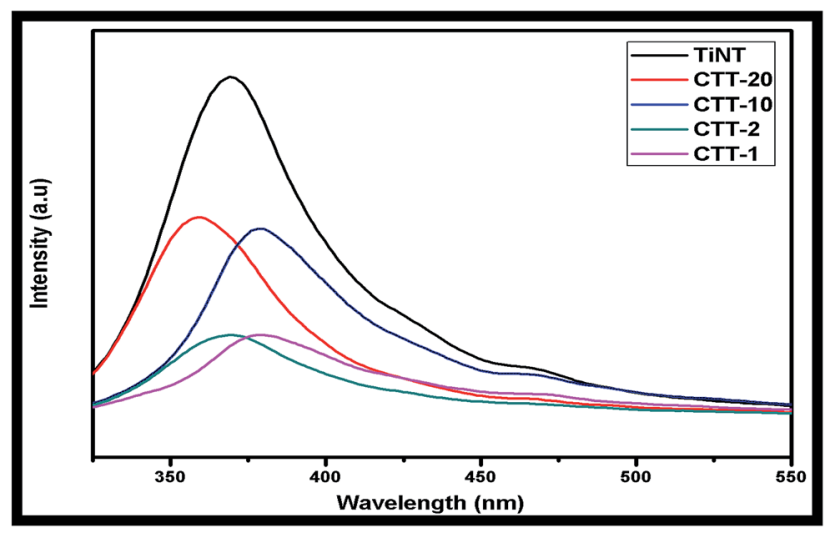

Fig. 6 PL spectra of TiNT, CTT-1, CTT-2, CTT-10 \& 20. 


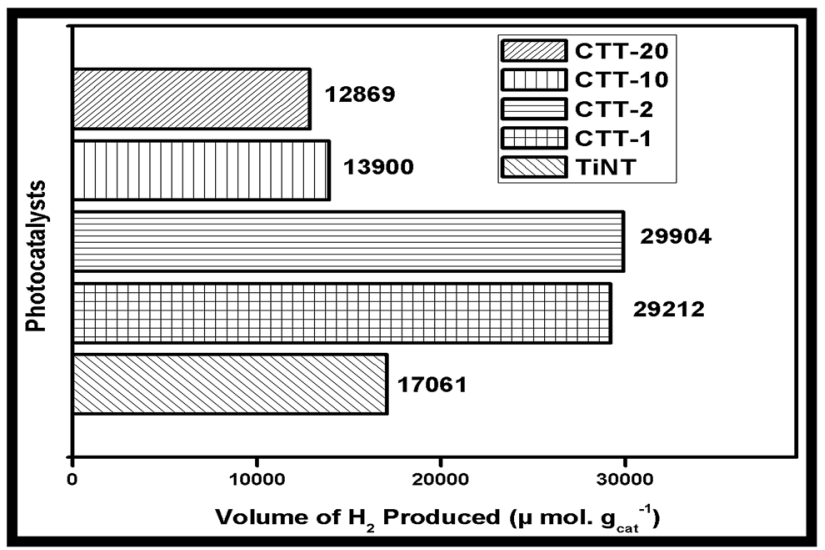

Fig. 7 Comparison of $\mathrm{H}_{2}$ production activity of TiNT, CTT-1, 2 \& 10 photocatalysts under solar irradiation for $4 \mathrm{~h}$.

the enhanced rate of $\mathrm{H}_{2}$ production under solar and visible light irradiation. ${ }^{\mathbf{1 0} c \boldsymbol{d} \boldsymbol{d}}$

\section{Structure activity correlation}

Based on the characterization results of the data it is evident that impregnation of FCNTs with TiNT's resulted in well dispersed catalysts and indicates strong interaction of FCNTs with TiNT. Electrons and holes get generated as the nanohybrid is exposed to sunlight in the conduction and valence bands respectively. Improvement in the visible light absorption and decreased band gap energy values of the nanohybrid could be due to the creation of intermediate energy levels of doped FCNTs. The possible photoexcitation and charge transfer processes are as shown in the Fig. 9. Lu et al., deduced that band gap is probably reduced as a result of doping state creation near to the $E_{\mathrm{F}}{ }^{10 e}$ The electrons accumulated on the conduction band of TiNT could be transferred to $\mathrm{H}^{+}$to produce $\mathrm{H}_{2}$ while holes in the valence band of TiNT are consumed by the sacrificial agent glycerol which subsequently gets mineralized leading to suppression of recombination of photogenerated charge carriers and enhancement of $\mathrm{H}_{2}$ production.

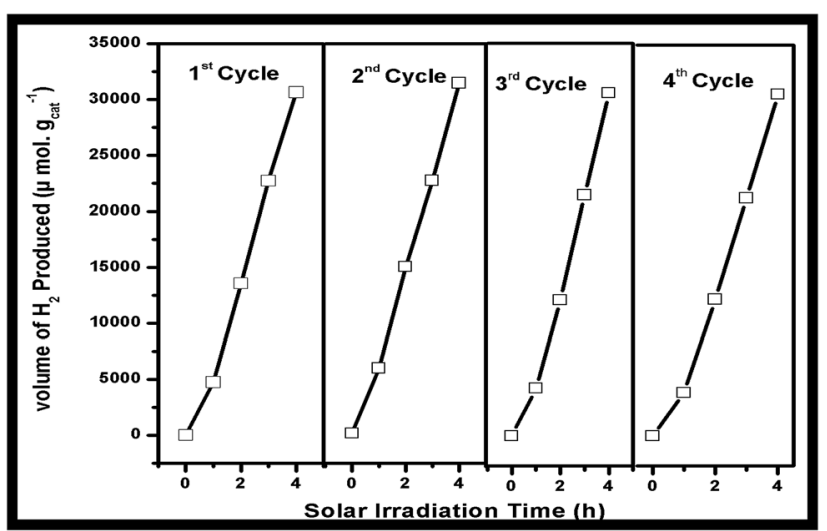

Fig. $8 \mathrm{H}_{2}$ production activity in 4 cycles using $5 \mathrm{mg} \mathrm{CTT-2}$ catalysts under solar irradiation.

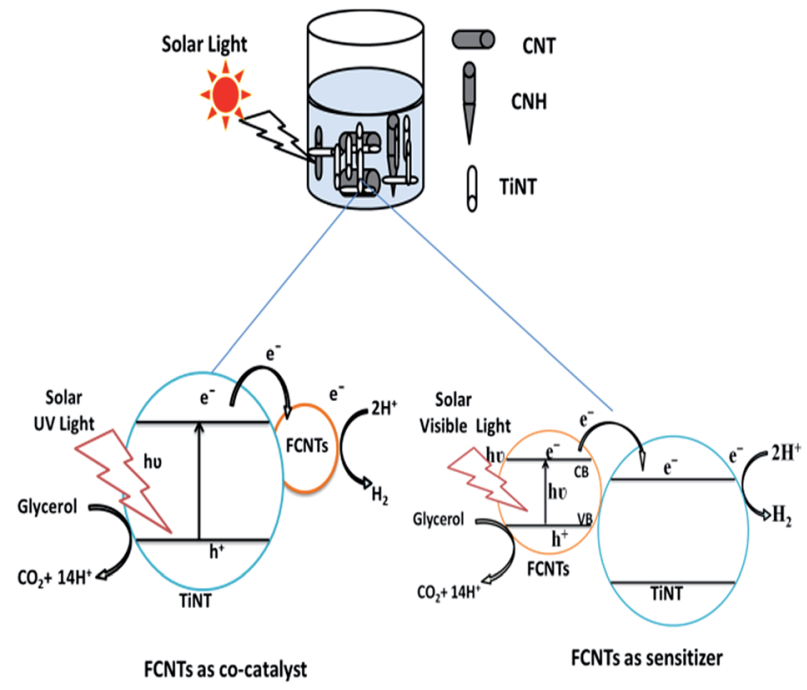

Fig. 9 Schematic illustration of mechanism of FCNTs under solar irradiation.

\section{Conclusions}

In conclusion this work resulted in synthesis of a series of novel functionalized carbon nanotube $/ \mathrm{TiO}_{2}$ nanotube nanohybrid materials by wet impregnation method to extend absorption to the visible region and also to retard the electron-hole pair recombination and thereby to enhance the $\mathrm{H}_{2}$ production capability under solar light irradiation. The percentage addition of FCNTs to TiNTs influences the photocatalytic activity of CTT composite. Highest photocatalytic $\mathrm{H}_{2}$ production was observed for CTT-2 photocatalysts mainly due to extension of absorption edge to the visible region and also by retardation of electronhole recombination as compared to other photocatalysts.

\section{Acknowledgements}

Financial support from University Grants Commission (UGC), New Delhi, India (F. No. 43-405/2014), is gratefully acknowledged.

\section{References}

1 (a) S. Ijima, Nature, 1991, 354, 56-58; (b) P. Rima, Z. Kumbhakara and A. K. Mitra, J. Mater. Sci. Eng. B, 2010, 167, 97-101; (c) A. Profumo, M. Fagnoni and D. Merli, J. Anal. Chem., 2006, 78, 4194-4199.

2 (a) K. Woan, G. Pyrgiotakis and W. Sigmund, Adv. Mater., 2009, 21, 2233-2239; (b) P. Serp and J. L. Figueiredo, Carbon Materials for Catalysis, Wiley, 2009, pp. 481-506; (c) Y. K. Kim and H. Park, Energy Environ. Sci., 2011, 4, 685-694. 3 (a) T. S. Natarajan, K. Natarajan, H. C. Bajaj and J. T. Rajesh, J. Nanopart. Res., 2013, 15(1669), 1-18; (b) G. Zhang, H. Huang, W. Li, F. Yu, H. Wu and L. Zhou, Electrochim. Acta, 2012, 81, 117-122; (c) Y. Qin, G. Wang and Y. Wang, Catal. Commun., 2007, 8, 926-930. 
4 (a) W. Wang, P. Serp, P. Kalck and J. L. Faria, Appl. Catal., B, 2005, 56, 305-312; (b) G. An, W. Ma, Z. Sun, Z. Liu, B. Han, Z. Miao and K. Ding, Carbon, 2007, 45, 1795-1801; (c) C. Song, P. Chen, C. Wang and L. Zhu, Chemosphere, 2012, 86, 853-859; (d) Y. Ou, J. Lin, S. Fang and D. Liao, Chem. Phys. Lett., 2006, 429, 199-203; (e) M. Mamatha Kumari, D. Praveen Kumar, P. Haridoss, V. Durga Kumari and M. V. Shankar, Int. J. Hydrogen Energy, 2014, 40, 1665-1674; (f) D. Praveen Kumar, N. Lakshmana Reddy, M. Mamatha Kumari, B. Srinivas, V. Durga Kumari, B. Sreedhar, V. Roddatis, O. Bondarchuk, M. Karthick, B. Nepolian and M. V. Shankar, Sol. Energy Mater. Sol. Cells, 2015, 136, 157166.

5 (a) C. G. Silva, M. J. Sampaio, R. R. Marques, L. A. Ferreira, P. B. Tavares, A. M. Silva and J. L. Faria, Appl. Catal., B, 2015, 178, 82-90; (b) G. D. Tarigh, F. Shemirani and N. S. Maz'hari, RSC Adv., 2015, 5, 35070-35079.

6 (a) S. Dong, H. Wang and Z. Wu, J. Phys. Chem. C, 2009, 113, 16717-16723; (b) R. A. Doong and L. F. Chiang, Water Sci. Technol., 2008, 58, 1985-1992.

7 (a) A. Miribangul, X. Ma, C. Zeng, H. Zou, Y. Wu, T. Fan and Z. Su, Photochem. Photobiol., 2016, 92, 523-527; (b) S. Xiao, W. Zhu, P. Liu, F. Liu, W. Dai, D. Zhang, W. Chen and H. Li, Nanoscale, 2016, 8, 2899-2907.

8 (a) K. M. Kim, H. O. Pae, M. Zheng, R. Park, Y. M. Kim and H. T. Chung, Circ. Res., 2007, 101, 919-927; (b) C. Lettmann, K. Hildenbrand, H. Kisch, W. Macyk and
W. F. Maier, Appl. Catal., B, 2001, 32, 215-227; (c) Y. Aoki, K. Urita, D. Noguchi, T. Itoh, H. Kanoh and T. Ohba, Chem. Phys. Lett., 2009, 482, 269-273; (d) M. Inagaki, M. Nonaka, F. Kojin, T. Tsumura and M. Toyoda, Environ. Sci. Technol., 2006, 27, 251-258; (e) Y. Xie, S. H. Heo, S. H. Yoo, G. Ali and S. O. Cho, Nanoscale Res. Lett., 2010, 5, 603-607.

9 (a) G. An, W. Ma, Z. Sun, Z. Liu, B. Han and S. Miao, Carbon, 2009, 47, 1795-1801; (b) C. Song, P. Chen, C. Wang and L. Zhu, Chemosphere, 2012, 86, 853-859; (c) S. K. Parayil, H. S. Kibombo, C. M. Wu, R. Peng, T. Kindle and J. Baltrusaitis, J. Phys. Chem. C, 2010, 117, 16850-16862; (d) K. Dai, T. Peng, D. Ke and B. Wei, Nanotechnology, 2009, 20, 125603-125609; (e) Y. Yu, J. C. Yu, J. Yu, Y. Kwok, Y. Che and J. Zhao, Appl. Catal., A, 2005, 289, 186-196.

10 (a) C. Hyo and B. Kim, Carbon, 2012, 50, 2472-2481; (b) X. J. Lv, S. X. Zhou, C. Zhang, H. X. Chang, Y. Chena and W. Fu, J. Mater. Chem., 2012, 22, 18542-18549; (c) D. Praveen Kumar, M. V. Shankar, M. Mamatha Kumari, G. Sadanandam, B. Srinivas and V. Durga Kumari, Chem. Commun., 2013, 49, 9443-9445; (d) K. Lalitha, G. Sadanandam, V. Durga Kumari, M. Subrahmanyam, B. Sreedhar and Y. Hebalkar Neha, J. Phys. Chem. C, 2010, 114, 22181-22189; (e) S. Y. Lu, C. W. Tang, Y. H. Lin, H. F. Kuo, Y. C. Lai, M. y. Tsai, H. Ouyang and K. Hsuw, Appl. Phys. Lett., 2010, 96, 231915-231923. 